\title{
THE SET WHERE AN APPROXIMATE DERIVATIVE IS A DERIVATIVE
}

\author{
RICHARD J. O'MALLEY
}

\begin{abstract}
Let $f:[0,1] \rightarrow R$ possess a finite approximate derivative $f_{\text {ap. }}^{\prime}$. Let $E$ be the set of points $x$ where $f$ is actually differentiable. It is shown that for every $\lambda$ if $\left\{x: f_{\text {ap }}^{\prime}(x)=\lambda\right\} \neq \varnothing$, then $\left\{x: f_{\text {ap }}^{\prime}(x)=\lambda\right\} \cap E \neq \varnothing$. A strengthening of the mean value theorem associated with approximate derivatives is an immediate corollary.
\end{abstract}

Introduction. In this paper we will be interested in functions $f:[0,1] \rightarrow R$ which possess a finite approximate derivative $f_{\text {ap. }}^{\prime}$. More precisely, we will investigate the set $E$ where $f$ is actually differentiable. Several facts are already known about $E$. For example in [1], C. Goffman and C. J. Neugebauer provide a simple proof that $E$ contains a dense open subset of $[0,1]$. Further, in [4], C. E. Weil develops two interesting properties of $E$. One property is that for every pair of numbers $a, b$ if $\left\{x: a<f_{\text {ap }}^{\prime}(x)<b\right\} \neq \varnothing$, then $\left\{x: a<f_{\text {ap }}^{\prime}(x)<b\right\}$ $\cap E \neq \varnothing$. Here, using methods not dependent on Weil's results, we establish a stronger property of $E$. Namely, for every real number $\lambda$, if $\left\{x: f_{\text {ap }}^{\prime}(x)=\lambda\right\}$ $\neq \varnothing$, then $\left\{x: f_{\text {ap }}^{\prime}(x)=\lambda\right\} \cap E \neq \varnothing$. This also shows that $f^{\prime}$ has the Darboux property on $E$ because $f_{\text {ap }}^{\prime}$ has the Darboux property. In turn, this leads to a strengthening of the mean value theorem associated with approximately differentiable functions.

We will use the following basic definitions and known properties: Let $m$ denote Lebesgue measure on $[0,1]$.

Definition 1 . A measurable set $A$ has density 1 at 0 if and only if $\lim _{x \rightarrow 0} m(A \cap[0, x]) / x=1$.

DEFINITION 2. A function $f$ is said to have an approximate derivative $f_{\text {ap }}^{\prime}$ on $[0,1]$ if for each $x_{0}$ in $[0,1]$ there is a set $A\left(x_{0}\right)$ having density 1 at 0 , such that $f\left(x_{0} \pm h\right)=f\left(x_{0}\right) \pm h\left(f_{\text {ap }}^{\prime}\left(x_{0}\right)+\lambda( \pm h)\right)$ where $\lim _{h \rightarrow 0} \lambda( \pm h)=0$ when $h$ is restricted to $A\left(x_{0}\right)$.

Property 1. The function $f_{\text {ap }}^{\prime}$ is a Baire class 1 function having the Darboux (intermediate value) property.

PROPERTY 2. If $f_{\text {ap }}^{\prime} \geqslant 0(\leqslant 0)$ on $(a, b) \subset[0,1]$, then $f$ is nondecreasing (nonincreasing), and $f_{\text {ap }}^{\prime}$ is the derivative of $f$ on $[a, b]$, one-sided at the endpoints. For further elaboration see [1] and [4].

We will need one lemma, the proof of which is straight-forward and differs very little from a lemma in Tolstoff [3, p. 499] or O'Malley [2, Lemma 3]. For brevity we have chosen to omit the details of the proof.

Received by the editors August 26, 1974.

AMS (MOS) subject classifications (1970). Primary 26A24.

Key words and phrases. Approximate derivative, Baire class 1, Darboux, density. 
Lemma. Let $f:[c, d] \rightarrow R$ possess an approximate derivative $f_{\text {ap. }}^{\prime}$ Let $\epsilon>0$ be fixed and $B(x)=\{y:|f(y)-f(x)|<\epsilon|y-x|\}$. Let $H_{n}$ be the set of those $x$ such that $m(B(x) \cap J)>\frac{1}{2} m(J)$ for all intervals $J \subset[c, d]$ with $x$ in $J$ and $m(J)<1 / n$. Then for the closure $\bar{H}_{n}$ of $H_{n}$ we have:

(a) If $x, y$ are in $\bar{H}_{n}$, with $|x-y|<1 / n$, then $|f(y)-f(x)| \leqslant \epsilon|y-x|$.

(b) If $x$ is in $\bar{H}_{n}$, then $m(B(x) \cap J) \geqslant \frac{1}{2} m(J)$ for all intervals $J \subset[c, d]$ with $x$ in $J$ and $m(J)<1 / n$.

We now prove our

THEOREM . Let $f:[0,1] \rightarrow R$ possess an approximate derivative $f_{\mathrm{ap}}^{\prime}$, and let $E=\left\{x: f^{\prime}\right.$ exists at $\left.x\right\}$. If $\lambda$ is any real number such that $\left\{x: f_{\mathrm{ap}}^{\prime}(x)=\lambda\right\} \neq \varnothing$, then $\left\{x: f_{\text {ap }}^{\prime}(x)=\lambda\right\} \cap E \neq \varnothing$.

Proof. It will suffice to let $\lambda=0$. In the general case we would then consider $g(x)=f(x)-\lambda x$. We let $\bar{G}$ denote the closure of $\left\{x: f_{\text {ap }}^{\prime}(x)=0\right\}$. By the Darboux property of $f_{\text {ap }}^{\prime}$ we have that, on any component interval of the complement of $\bar{G}, f_{\text {ap }}^{\prime}$ is of constant sign. Hence by Property $2, f$ is strictly monotonic and differentiable on the closure of each such component, onesided at the endpoints. In turn, this assures us that $f^{\prime}$ exists and equals zero at any isolated point $x_{0}$ of $\bar{G}$. We therefore need only consider the case where $\bar{G}$ is perfect.

Let $I$ be any open interval having nonempty intersection with $\bar{G}$, and let $\epsilon>0$ be fixed. We prove that it is possible to find a closed interval $[c, d] \subset I$ such that

(1) $(c, d) \cap \bar{G} \neq \varnothing$, and

(2) $|f(y)-f(x)| \leqslant 2 \epsilon|y-x|$ for all $x$ in $[c, d] \cap \bar{G}$ and $y$ in $[c, d]$.

This will establish the theorem, for we need only consider a sequence of $\epsilon_{k}$ strictly decreasing to zero and an associated sequence of closed intervals $\left[a_{k}, b_{k}\right]$ such that

(3) $\left[a_{k+1}, b_{k+1}\right] \subset\left(a_{k}, b_{k}\right)$,

(4) $\left(a_{k}, b_{k}\right) \cap \bar{G} \neq \varnothing$, and

(5) $|f(y)-f(x)| \leqslant 2 \epsilon_{k}|y-x|$ for all $x$ in $\left[a_{k}, b_{k}\right] \cap \bar{G}$ and $y$ in $\left[a_{k}, b_{k}\right]$.

The intersection of the sequence of sets $\bar{G} \cap\left[a_{n}, b_{n}\right]$ will be nonempty, and at any $x_{0}$ in this intersection $f^{\prime}$ exists and equals zero.

Since $f_{\text {ap }}^{\prime}$ is a Baire class 1 function and $\bar{G}$ is a perfect set, the function $f_{\text {ap }}^{\prime}$ has a point of continuity relative to $\bar{G}$ in $I \cap \bar{G}$. Since $\left\{x: f_{\text {ap }}^{\prime}(x)=0\right\}$ is dense in $\bar{G}, f_{\text {ap }}^{\prime}=0$ at any such point of continuity. Hence for the $\epsilon$ given above we may find a closed subinterval of $I, I_{1}=\left[c_{1}, d_{1}\right]$, whose endpoints are bilateral limit points of $\bar{G}$, such that $\left|f_{\text {ap }}^{\prime}(x)\right|<\epsilon$ for all $x$ in $I_{1} \cap \bar{G}$. For this $I_{1}$ and $\epsilon>0$ we define $B(x)$ and $H_{n}$ as in the lemma. From Definition 1 and the fact that $\left|f_{\text {ap }}^{\prime}(x)\right|<\epsilon$ for all $x$ in $I_{1} \cap \bar{G}$, it follows that $\cup_{n=1}^{\infty}\left(\bar{H}_{n} \cap \bar{G}\right)$ $=\bar{G} \cap I_{1}$. By the Baire category theorem there is an $N$ and an interval $(c, d)$ with $c_{1}<c<d<d_{1}$ such that $\varnothing \neq(c, d) \cap \bar{G} \subset \bar{H}_{N} \cap \bar{G}$. Further, we may choose $(c, d)$ so that $0<d-c<1 / N$ and $c$ and $d$ are bilateral limit points of $\bar{G}$. Then by the lemma we have:

(6) If $x$ and $y$ belong to $[c, d] \cap \bar{G}$, then $|f(x)-f(y)| \leqslant \epsilon|y-x|$.

(7) If $x$ belongs to $[c, d] \cap \bar{G}$ and $J$ is a subinterval of $[c, d]$ containing $x$, then $m(B(x) \cap J) \geqslant \frac{1}{2} m(J)$. 
Let $(a, b) \subset[c, d]$ be any component interval of the complement of $\bar{G}$. The function $f$ is strictly monotone on $[a, b]$, and it will cause no loss of generality to suppose that it is strictly increasing. By $(6), f(b)-f(a) \leqslant \epsilon(b-a)$. Hence, if for some $y_{0}$ in $(a, b)$ there is a $\gamma>0$ for which

$$
f\left(y_{0}\right)-f(a)>2(1+\gamma) \epsilon\left(y_{0}-a\right),
$$

we must have that $2(1+\gamma)\left(y_{0}-a\right)+a=z_{0}<b$ and $f(y)-f(a)>\epsilon(y-a)$ for all $y$ in $\left[y_{0}, z_{0}\right]$. However, this implies that $m\left(B(a) \cap\left[a, z_{0}\right]\right) \leqslant y_{0}-a<$ $1 / 2 m\left(\left[a, z_{0}\right]\right)$, contradicting (7). This contradiction proves that $f(y)-f(a) \leqslant$ $2 \epsilon(y-a)$ for all $y$ in $[a, b]$. In the same fashion we can prove that, for all $y$ in $[a, b], f(b)-f(y) \leqslant 2 \epsilon(b-y)$.

We are now ready to show that $[c, d]$ satisfies (2). It is clear that $[c, d]$ satisfies (1). Let $x$ belong to $[c, d] \cap \bar{G}$ and $y$ to $[c, d]$. If $y$ also belongs to $\bar{G}$ then $|f(y)-f(x)| \leqslant \epsilon|y-x|$. If $y$ does not belong to $\bar{G}$, there is a component interval of the complement, $(a, b) \subset[c, d]$, to which $y$ belongs. Then, assuming without loss of generality that $x \leqslant a<y$, we have:

$$
|f(x)-f(a)| \leqslant \epsilon|x-a|=\epsilon(a-x),
$$

and

$$
|f(y)-f(a)| \leqslant 2 \epsilon|y-a|=2 \epsilon(y-a),
$$

so $|f(x)-f(y)| \leqslant 2 \epsilon|x-y|$. This proves that (2) is satisfied and, as was mentioned after (2), is enough to establish the theorem.

Corollary 1. Let $f:[0,1] \rightarrow R$ have an approximate derivative $f_{\text {ap }}^{\prime}$. Let $E=\left\{x: f^{\prime}\right.$ exists at $\left.x\right\}$. Then $f^{\prime}$ has the Darboux property on $E$.

Proof. This is obvious since $f_{\text {ap }}^{\prime}$ has the Darboux property.

Corollary 2. Let $f:[0,1] \rightarrow R$ have an approximate derivative $f_{\text {ap. Then }}^{\prime}$ there is a point $x_{0}$ in $(0,1)$ at which $f$ is differentiable such that $f(1)-f(0)$ $=f^{\prime}\left(x_{0}\right)$.

Proof. In [1] it is shown that there is an $x_{1}$ in $(0,1)$ such that $f(1)-f(0)$ $=f_{\mathrm{ap}}^{\prime}\left(x_{1}\right)$. Hence $\left\{x: f_{\mathrm{ap}}^{\prime}(x)=f(1)-f(0)\right\} \neq \varnothing$.

\section{REFERENCES}

1. C. Goffman and C. J. Neugebauer, On approximate derivatives, Proc. Amer. Math. Soc. 11 (1960), 962-966. MR 22 \#9562.

2. R. J. O’Malley, A density property with applications, Trans. Amer. Math. Soc. 199 (1974), $75-87$.

3. G. Tolstoff, Sur la dérivée approximative exacte, Mat. Sb. 4 (1938), 499-504.

4. C. E. Weil, On approximate and Peano derivatives, Proc. Amer. Math. Soc. 20 (1969), 487 490. MR $38 \# 2265$.

Department of Mathematics, University of Wisconsin, Milwaukee, Wisconsin 53201 